\title{
EXTRAÇÃO E DETERMINAÇÃO DO TEOR SOLÚVEL DE ZINCO DO SOLO *
}

\author{
A. O. JaCINTHO \\ R. A. Catani ** \\ A. Pizzinatto
}

Foram estudadas diversas soluções extratoras e várias técnicas para extração do zinco solúvel do solo.

Foram utilizadas cinco amostras de solos do Estado de São Paulo e as determinações foram feitas empregando-se o método baseado na espectrofotometria de chama de absorção atômica.

Para extração do zinco solúvel foram empregadas as seguintes soluções: $\mathrm{HCl} 0,05$ e $0,10 \mathrm{~N} ; \mathrm{CH}_{3} \mathrm{COOH} 0,10 \mathrm{~N}$; EDTA dissódico a $1 \% ; \mathrm{MgCl}_{2}$ 0,10 e $0,50 \mathrm{~N} ; \mathrm{H}_{2} \mathrm{SO}_{4} 0,05$ e $0,10 \mathrm{~N}$. As extrações com as soluções de $\mathrm{HCl}$, $\mathrm{CH}_{: 3} \mathrm{COOH}$, EDTA dissódico e $\mathrm{MgCl}_{2}$, foram conduzidas por agitação de 2,5 e $5,0 \mathrm{~g}$ de solo com $50 \mathrm{ml}$ de solução, durante 10 , 15 e 30 minutos.

Com as soluções de $\mathrm{H}_{2} \mathrm{SO}_{4}$, as extrações foram feitas apenas a partir de 5,0 g de solo para $50 \mathrm{ml}$ de solução, conservando-se as demais condições.

Os dados obtidos revelaram que de um modo geral as soluções de EDTA a $1 \%$, de $\mathrm{HCl} 0,05$ e $0,10 \mathrm{~N}$, e de $\mathrm{H}_{2} \mathrm{SO}_{4} 0,05$ e $0,10 \mathrm{~N}$, foram mais eficientes na extração do zinco do que as soluções de $\mathrm{MgCl}_{2} 0,10$ e $0,50 \mathrm{~N}$ e de $\mathrm{CH}_{3} \mathrm{COOH} 0,10 \mathrm{~N}$.

O tempo de agitação para extração do zinco durante 15 minutos forneceu resultados mais elevados (significativos ao nível de 5\% de probabilidade) do que os obtidos com 10 minutos. No entanto a agitação durante 30 minutos apresentou resultados que não diferiram dos conseguidos com 15 minutos.

A proporção de 2,5:50 (pêso da terra em gramas para volume em $\mathrm{ml}$ de solução extratora) apresentou resultados mais elevados, e significativos ao nível de 5\%, do que os obtidos com a proporção de 5:50.

* Entregue para publicação em 23-12-1971.

*: Departamento de Química da E.S.A. "Luiz de Queiroz" U.S.P.

**: Bolsista da Fundação de Amparo à Pesquisa do Estado de S. Paulo.

$*:: *$ Agradecimentos ao Departamento de Matemática e Estatística da ESALQ pela análise estatistica dos dados. 


\section{INTRODUÇÃO}

A determinação do zinco do solo, quando extraído por diversas soluções, tem sido executada por muitos autores, através de diversas técnicas.

BROW, KRANTZ \& MARTIN (1962; 1964), utilizaram os teores de zinco extraídos de diversos solos, com solução de acetato de amônio contendo ditizona para correlacionar coml as respostas às aplicações de zinco e com os sintomas de deficiência em várias plantas.

MARTENS \& CHESTERS (1967) concluiram que a absorção do zinco do solo pelas plantas poderia ser estabelecida de uma forma mais adequada, através de uma equação de regressão múltipla na qual figurariam diversas variáveis independentes, além do teor de zinco extraído com solução $0,1 \mathrm{~N}$ de HC1.

MARTENS (1968) concluiu que o zinco extraído com solução $2 \mathrm{~N}$ de $\mathrm{MgC1}_{2}$ apresenta melhor correlação com zinco absorvido pelas plantas, para solos de características variáveis, confirmando os dados obtidos por STEWART \& BERGER (1965).

O zinco pode ocorrer no solo adsorvido aos colóides, além de em outras formas, podendo ser extraído por diversas soluções e diferentes técnicas (CHAPMAN, 1966; STEWART \& BERGER, 1965; VIETS, JR. \& BOAWN, 1965; JACKSON, 1958).

Ainda que o teor de zinco extraído com solução de acetato de amônio e o solúvel em água, tenham sido considerados disponíveis às plantas, na realidade pouco se sabe sôbre a disponibilidade do referido elemento quando extraído com as mencionadas soluções e outras e em condições variáveis de extração (VIETS JR. \& BOAWN 1965; SORENSEN, DELSLIGLE \& KNUNDSEN, 1971; BROWN, QUINCK \& EDDINGSS 1971).

Quanto ao teor total, o zinco do solo pode variar, segundo CHAPMAN (1966), de 10 a 30 ppm, enquanto HODGSON (1963), apresentou dados médios oscilando entre 40 e $58 \mathrm{ppm}$. -

Como para as condições do Estado de São Paulo, poucos são os dados referentes ao zinco do solo, principalmente devido às dificuldades analíticas, considerou-se oportuno um estudo sobre a solubilidade do referido elemento em diferentes soluções extratoras. As dificuldades analíticas foram superadas pelo emprego da espectrofotometria de absorção atômica.

O presente trabalho foi realizado com o objetivo de contribuir para o estabelecimento de métodos e técnicas convenientes, de extração e determinação do teor solúvel de zinco do solo em diversas soluções extratoras. 


\section{MATERIAL E MÉTODOS}

O material constituiu-se de 5 amostras (horizonte Ap) dos solos adiante discriminados:

\begin{tabular}{cll}
\hline A m o s t a s & \multicolumn{1}{c}{$\mathbf{S} \mathbf{o} \mathbf{~ o}$} \\
1 & Latosólico roxo 1 \\
2 & Latosólico roxo 2 \\
3 & Podzólico de Lins e Marília, Var. Lins \\
4 & Podzólico Vermelho Amarelo - orto. \\
5 & Latosólico Vermelho Amarelo fase arenosa
\end{tabular}

As determinações do zinco foram executadas pelo método baseado na espectrofotometria de chama de absorção atômica; por sua simplicidade e rapidez. Empregou-se o Espectrofotômetro de Absorção Atômica Perkim Elmer, modêlo 303.

\section{Reativos}

Solução estoque n.․ 1 de zinco (1.000 ppm). Dissolveram-se 250 , mg de zinco metálico em $5 \mathrm{ml}$ de ácido clorídrico $\pm 6 \mathrm{~N}$ (destilado), transferiu-se para balão volumétrico de $250 \mathrm{ml}$ e completou-se o volume com água desmineralizada.

Solução estoque.$^{\circ} 2$ de zinco $(10 \mathrm{ppm})$. Preparada através de diluição da solução estoque.

Soluções de HC1 0,05 e 0,10 N. Preparadas a partir da diluição do ácido concentrado destilado e, posteriormente, aferidas.

Soluções de $\mathrm{CH}_{3} \mathrm{COOH}$ 1,00 e 0,10 N. Preparadas a partir do ácido acético glacial, p.a. Baker, através de diluição e, em seguida, aferidas.

Solução de EDTA dissódico a 1\%. Dissolveram-se $10 \mathrm{~g}$ do sal contendo 2 moléculas de água de cristalização (titriplex III - Merck) em água desmineralizada e completou-se o volume a 1 litro.

Solução de $\mathrm{MgC1}_{2}$ 2,0 N. Dissolveram-se 203,33 g do sal contendo 6 moléculas de água de cristalização, p.a. Baker, em água desmineralizada e completou-se o volume a 1 litro.

Soluções de $\operatorname{MgC1}_{2} 0,10$ e 0,50 N. Preparadas através de diluição da solução de $\mathrm{MgC1}_{2} 2,0 \mathrm{~N}$. 
Solução de $\mathrm{H}_{2} \mathrm{SO}_{4}$ 0,05 e 0,10 N. Preparadas através de diluição de $\mathrm{H}_{2} \mathrm{SO}_{4}$ concentrado p.a. Baker e, posteriormente, aferidas.

As soluções foram conservadas em frascos plásticos.

\section{Estabelecimento das equações de regressão para a determinação de zinco nas diversas soluções extratoras}

Procedimento: Foram preparadas soluções padrões contendo 0,2 $-0,4-0,6-0,8$ e $1,0 \mathrm{ppm}$, a partir da solução estoque $n .^{\circ} 2$ de zinco $(10 \mathrm{ppm})$, em cada uma das soluções extratoras.

Posteriormente foram feitas as leituras ( 5 repetições para cada padrão) no espectrofotômetro de absorção atômica e com os resultados foram estabelecidas as seguintes equações de regressão:

$\mathbf{X}=(\mathbf{A}+1,29) 0,030$, para a solução de $\mathrm{HC1} \quad 0,05 \mathrm{~N}$

$X=(A+0,79) 0,031$, para a solução de $\mathrm{HCl} 0,10 \mathrm{~N}$

$X=(A+1,03) 0,031$, para a solução de $\mathrm{CH}_{3} \mathrm{COOH} 0,10 \mathrm{~N}$

$X=(A+0,56) 0,031$, para a solução de EDTA dissódico a $1 \%$

$\mathrm{X}=(\mathrm{A}+0,55) 0,035$, para a solução de $\mathrm{MgC1}_{2} 0,10 \mathrm{~N}$

$\mathrm{X}=\left(\mathrm{A}+\mathbf{0 , 4 6 )} 0,038\right.$, para a solução de $\mathrm{MgC1}_{2} \mathbf{0 , 5 0 \mathrm { N }}$

$X=(A+0,53) 0,033$, para a solução de $\mathrm{H}_{2} \mathrm{SO}_{4} 0,05 \mathrm{~N}$

$\mathrm{X}=(\mathrm{A}+0,54) 0,034$, para a solução de $\mathrm{H}_{2} \mathrm{SO}_{4} 0,10 \mathrm{~N}$

Nas equações 1 a 8, A representa a absorbância multiplicada por 100 ; e X microgramas de zinco ( $\mathrm{Zn})$ por $\mathrm{ml}$ ou ppm de $\mathrm{Zn}$ na solução.

\section{Preparo das amostras de solos}

As amostras, devidamente coletadas para análise de micronutrientes, foram sêcas ao ar, peneiradas através de peneira de plástico de malha fina e, em seguida, moidas em gral de ágata.

\section{Preparo dos extratos}

Transferiram-se 2,5 e ou 5,0 g de solo para frasco de plástico de $500 \mathrm{ml}$ e adicionaram-se $50 \mathrm{ml}$ de solução extratora. Colocou-se em agitação durante 10 ou 15 ou 30 minutos, filtrou-se através de papel de filtro Whatman $n .^{\circ} 1$, refiltrando-se a primeira porção, obtendo-se assim o extrato.

\section{Precisão e exatidão do método}

A precisão do método baseado na fotometria de absorção atômica foi avaliada através da determinação do zinco adicionado em cinco concentrações diferentes na forma de soluções padrões aos diversos extratores. $O$ cálculo do valor médio do coeficiente de variação forneceu uma indicação da precisão do método.

A exatidão do método foi avaliada, através de um ensaio de recuperação, apenas para extração com solução de $\mathrm{HC} 10,10 \mathrm{~N}$ na proporção de $5 \mathrm{~g}$ de solo para $50 \mathrm{ml}$ de solução HC1. 


\section{Determinação do zinco nos diversos extratos}

Os extratos preparados foram levados diretamente para o aparelho Perkim Elmer 303 tendo sido feitas 3 a 5 leituras em cada extrato. Simultaneamente foram executadas determinações de, pelo menos dois padrões, nas respectivas soluções extratoras.

\section{RESULTADOS OBTIDOS E DISCUSSÃO}

Os resultados obtidos são apresentados nos quadros n. ${ }^{0} 1$ a 8 .

\section{Precisão e exatidão do método}

Os dados obtidos referentes a avaliação da precisão do método, fundamentado na espectrofotometria de absorção atômica acham-se reunidos no quadro 1 .

QUADRO 1 - Valores dos coeficientes de variação médios, obtidos para cinco concentrações de zinco adicionado na forma de solução padrão, com as diferentes soluções extratoras.

Soluções Extratoras
Coeficiente de variação

(médio)

$\begin{array}{rcccc}\mathrm{MgC1}_{2} & 0,10 & \mathrm{~N} & \% \\ \mathrm{MgCl}_{2} & 0,50 & \mathrm{~N} & 0,86 \\ \mathrm{CH}_{3} \mathrm{COOH} & 0,10 & \mathrm{~N} & 0,55 \\ \text { EDTA. } \mathrm{Na} & \mathrm{a} & 1 \% & 1,23 \\ \mathrm{HCl} & 0,05 & \mathrm{~N} & 0,73 \\ \mathrm{HC1} & 0,10 & \mathrm{~N} & 4,47 \\ \mathrm{H}_{2} \mathrm{SO}_{4} & 0,05 & \mathrm{~N} & 5,72 \\ \mathrm{H}_{2} \mathrm{SO}_{4} & 0,10 & \mathrm{~N} & 0,75 \\ \end{array}$

Os valores obtidos para o coeficiente de variação evidenciam que o método apresenta precisão satisfatória.

A exatidão do método, conforme foi esclarecido, foi apreciada através de ensaio de recuperação realizado em extratos de alguns solos, quando se empregou como extrator a solução de HC1 $0,10 \mathrm{~N}$ e com o tempa de extração de 15 minutos na proporção de $5,0 \mathrm{~g}$ de solo para $50 \mathrm{ml}$ de solução. Foram utilizados $5 \mathrm{ml}$ dos extratos dos solos n.os 2, 3 e 4 . Os resultados constam do quadro 2 . 
QUADRO 2 - Resultados do ensaio de recuperação feito com $5 \mathrm{ml}$ de extratos com solos 2,3 e 4 , obtidos na proporção de $5 \mathrm{~g}$ de solo para

$50 \mathrm{ml}$ de $\mathrm{HC} 10,10 \mathrm{~N}$ e com o tempo de agitação de 15 minutos.

\begin{tabular}{c|c|c|c|c|c}
\hline $\begin{array}{c}\text { Solo } \\
\text { n. }\end{array}$ & No extrato & Adicionado & Calculado & Encontrado & $\begin{array}{c}\text { Recupera- } \\
\text { ção (\%) }\end{array}$ \\
\cline { 2 - 5 } 2 & 0,25 & 0,40 & 0,65 & 0,67 & 103 \\
3 & 0,15 & 0,40 & 0,55 & 0,57 & 103 \\
4 & 0,12 & 0,40 & 0,52 & 0,54 & 104 \\
\hline
\end{tabular}

Os dados do quadro 2 permitem concluir que a exatidão do método, avaliada pelo ensaio de recuperação e nas condições descritas, é satisfatória.

\section{Extração e determinação do zinco}

Observando-se os dados do quadro 3, verifica-se que as soluções de EDTA e HC1 apresentaram pràticamente a mesma capacidade de extração de zinco nas amostras estudadas. As soluções de $\mathrm{MgC1}_{2}$ e $\mathrm{CH}_{3} \mathrm{COOH}$ extraíram menores quantidades de zinco, revelando-se o $\mathrm{MgC1}_{2}$ como o extrator menos eficiente.

QUADRO 3 - Teores de zinco solúvel (em ppm) nos diferentes solos. Extrações executadas na proporção de $2,5 \mathrm{~g}$ de solo para $50 \mathrm{ml}$ de várias soluções extratoras com 10 minutos de apitação.

\begin{tabular}{|c|c|c|c|c|c|c|}
\hline \multirow{3}{*}{$\begin{array}{c}\text { Amostras } \\
\text { de solo } \\
\text { N. }{ }^{\circ}\end{array}$} & \multicolumn{6}{|c|}{ Soluções extratoras } \\
\hline & \multicolumn{2}{|c|}{$\mathrm{MgCl}_{2}$} & \multirow{2}{*}{$\frac{\mathrm{CH}_{3} \mathrm{COOH}}{0,10 \mathrm{~N}}$} & \multirow{2}{*}{ 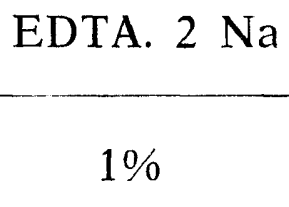 } & \multicolumn{2}{|c|}{$\mathrm{HCl}$} \\
\hline & $0,10 \mathrm{~N}$ & $0,50 \mathrm{~N}$ & & & $0,05 \mathrm{~N}$ & $0,10 \mathrm{~N}$ \\
\hline & \multicolumn{2}{|c|}{$\mathrm{ppm} \mathrm{Zn}$} & ppm Zn & ppm Zn & \multicolumn{2}{|c|}{ ppm Zn } \\
\hline 1 & 1,1 & 1,6 & 2,1 & 5,0 & 5,2 & 5,4 \\
\hline 2 & 0,9 & 1,7 & 2,5 & 4,6 & 4,5 & 4,6 \\
\hline 3 & 0,9 & 1,2 & 1,7 & 2,8 & 3,3 & 3,2 \\
\hline 4 & 0,9 & 1,2 & 1,7 & 2,3 & 2,4 & 2,4 \\
\hline 5 & 1,2 & 1,2 & 1,7 & 1,6 & 2,0 & 2,0 \\
\hline
\end{tabular}


QUADRO 4 - Teores de zinco solúvel (em ppm) nos diferentes solos. Extrações executadas na proporção de $2,5 \mathrm{~g}$ de solo para $50 \mathrm{ml}$ de várias soluçỗes extratoras com 15 minutos de agitação.

\begin{tabular}{|c|c|c|c|c|c|c|}
\hline \multirow{3}{*}{$\begin{array}{c}\text { Amostras } \\
\text { de solo } \\
\text { N. }\end{array}$} & \multicolumn{6}{|c|}{ Soluções extratoras } \\
\hline & \multicolumn{2}{|c|}{$\mathrm{MgC1}_{2}$} & \multirow{2}{*}{$-\frac{\mathrm{CH}_{3} \mathrm{COOH}}{0,10 \mathrm{~N}}$} & \multirow{2}{*}{$\frac{\text { EDTA. } 2 \mathrm{Na}}{1 \%}$} & \multicolumn{2}{|c|}{$\mathrm{HC1}$} \\
\hline & $0,10 \mathrm{~N}$ & $0,50 \mathrm{~N}$ & & & $0,05 \mathrm{~N}$ & $0,10 \mathrm{~N}$ \\
\hline & \multicolumn{2}{|c|}{ ppm $\mathrm{Zn}$} & ppm Zn & ppm Zn & \multicolumn{2}{|c|}{ ppm Zn } \\
\hline 1 & 1,2 & 1,4 & 2,4 & 6,3 & 5,7 & 5,6 \\
\hline 2 & 0,9 & 1,7 & 2,6 & 5,8 & 5,3 & 5,1 \\
\hline 3 & 0,9 & 1,4 & 1,8 & 3,2 & 3,4 & 3,7 \\
\hline 4 & 1,1 & 1,1 & 1,8 & 2,7 & 2,8 & 2,4 \\
\hline 5 & 1,2 & 1,2 & 1,8 & 1,8 & 2,2 & 2,1 \\
\hline
\end{tabular}

As soluções de $\mathrm{HC} 10,05$ e $0,10 \mathrm{~N}$ forneceram resultados praticamente iguais entre si, porém não diferindo estatisticamente dos obtidos com solução a $1 \%$ de EDTA.

Teores bem menores foram solubilizados pelas soluções de $\mathrm{MgC1}_{2}$ e $\mathrm{CH}_{3} \mathrm{COOH}$, embora o $\mathrm{CH}_{3} \mathrm{COOH}$ tenha se mostrado um pouco mais eficiente.

QUADRO 5 - Teores de zinco solúvel (em ppm) nos diferentes solos. Extrações executadas na proporção de $2,5 \mathrm{~g}$ de solo para $50 \mathrm{ml}$ de várias soluções extratoras, com 30 minutos de agitação.

\begin{tabular}{|c|c|c|c|c|c|c|}
\hline \multirow{3}{*}{$\begin{array}{c}\text { Amostras } \\
\text { de solo } \\
\text { N. }\end{array}$} & \multicolumn{6}{|c|}{ Soluções extratoras } \\
\hline & \multicolumn{2}{|c|}{$\mathrm{MgC1}_{2}$} & \multirow{2}{*}{$\frac{\mathrm{CH}_{3} \mathrm{COOH}}{0,10 \mathrm{~N}}$} & \multirow{2}{*}{$\frac{\text { EDTA. } 2 \mathrm{Na}}{1 \%}$} & \multicolumn{2}{|c|}{$\mathrm{HC1}$} \\
\hline & $0,10 \mathrm{~N}$ & $0,50 \mathrm{~N}$ & & & $0,05 \mathrm{~N}$ & $0,10 \mathrm{~N}$ \\
\hline & \multicolumn{2}{|c|}{ ppm Zn } & ppm Zn & ppm Zn & \multicolumn{2}{|c|}{ ppm Zn } \\
\hline 1 & 1,0 & 1,3 & 2,7 & 5,0 & 6,0 & 5,8 \\
\hline 2 & 0,9 & 1,2 & 3,0 & 4,4 & 5,7 & 5,2 \\
\hline 3 & 1,8 & 1,5 & 1,8 & 2,5 & 3,8 & 3,5 \\
\hline 4 & 1,1 & 1,3 & 1,8 & 2,1 & 2,8 & 2,9 \\
\hline $5^{\circ}$ & 1,2 & 1,4 & 1,6 & 1,4 & 2,3 & 2,4 \\
\hline
\end{tabular}


Os dados do quadro 5 permitem observar que os teores mais elevados foram obtidos com as soluções de $\mathrm{HC1}$ e de EDTA.

Os teores mais baixos foram extraídos, pelas soluções de $\mathrm{CH}_{3} \mathrm{COOH} 0,10 \mathrm{~N}, \mathrm{MgC1}_{2}$ 0,50 $\mathrm{N}$ e $\mathrm{MgC1}_{2} 0,10 \mathrm{~N}$, confirmando os dados dos quadro 3 e 4.

QUADRO 6 - Teores de zinco solúvel (em ppm) nos diferentes solos. Extracões executadas na proporção de $5,0 \mathrm{~g}$ de solo para $50 \mathrm{ml}$ de várias soluções extratoras com 10 minutos de agitação.

\begin{tabular}{|c|c|c|c|c|c|c|c|c|}
\hline \multirow{3}{*}{ 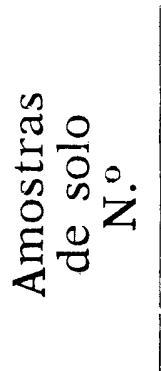 } & \multicolumn{8}{|c|}{ Soluções extratoras } \\
\hline & \multicolumn{2}{|c|}{$\mathrm{MgC1}_{2}$} & \multirow{2}{*}{$\frac{\mid \mathrm{CH}_{3} \mathrm{COOH}}{0,10 \mathrm{~N}}$} & \multirow{2}{*}{$\frac{\text { EDTA. } 2 \mathrm{Na}}{1 \%}$} & \multicolumn{2}{|c|}{$\mathrm{HCl}$} & \multicolumn{2}{|c|}{$\mathrm{H}_{2} \mathrm{SO}_{4}$} \\
\hline & $0,10 \mathrm{~N}$ & $0,50 \mathrm{~N}$ & & & $0,05 \mathrm{~N}$ & $0,10 \mathrm{~N}$ & $0,05 \mathrm{~N}$ & $0,10 \mathrm{~N}$ \\
\hline & \multicolumn{2}{|c|}{ ppm Zn } & \multicolumn{2}{|c|}{$\mathrm{ppm} \mathrm{Zn}$} & \multicolumn{2}{|c|}{ ppm Zn } & \multicolumn{2}{|c|}{ ppm Zn } \\
\hline 1 & 0,5 & 0,7 & 1,2 & 4,3 & 4,5 & 5,3 & 4,7 & 4,7 \\
\hline 2 & 0,5 & 0,9 & 1,4 & 3,9 & 4,4 & 4,7 & 4,2 & 4,4 \\
\hline 3 & 0,5 & 1,4 & 0,9 & 2,4 & 2,6 & 2,9 & 2,9 & 2,6 \\
\hline 4 & 0,5 & 1,0 & 0,9 & 1,8 & 2,1 & 2,3 & 2,0 & 1,7 \\
\hline 5 & 1,0 & 1,1 & 1,0 & 1,3 & 1,6 & 1,7 & 1,5 & 1,5 \\
\hline
\end{tabular}

QUADRO 7 - Teores de zinco solúvel (em ppm) nos diferentes solos. Extrações executadas na proporção de $5,0 \mathrm{~g}$ de solo para $50 \mathrm{ml}$ de várias soluçỗes extratoras, com 30 minutos de agitação.

\begin{tabular}{|c|c|c|c|c|c|c|c|c|}
\hline \multirow{3}{*}{ 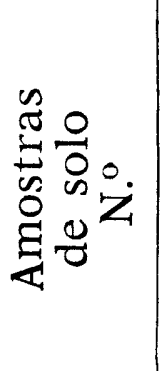 } & \multicolumn{8}{|c|}{ Soluções extratoras } \\
\hline & \multicolumn{2}{|c|}{$\mathrm{MgC1}_{2}$} & \multirow{2}{*}{$\frac{\mid \mathrm{CH}_{3} \mathrm{COOH}}{0,10 \mathrm{~N}}$} & \multirow{2}{*}{$\frac{\mid \text { EDTA. } 2 \mathrm{Na} \mid}{1 \%}$} & \multicolumn{2}{|c|}{$\mathrm{HCl}$} & \multicolumn{2}{|c|}{$\mathrm{H}_{2} \mathrm{SO}_{4}$} \\
\hline & $0,10 \mathrm{~N}$ & $0,50 \mathrm{~N}$ & & & $0,05 \mathrm{~N}$ & $0,10 \mathrm{~N}$ & $0,05 \mathrm{~N}$ & $0,10 \mathrm{~N}$ \\
\hline & \multicolumn{2}{|c|}{ ppm Zn } & \multicolumn{2}{|c|}{ ppm Zn } & \multicolumn{2}{|c|}{$\mathrm{ppm} \mathrm{Zn}$} & \multicolumn{2}{|c|}{ ppm $\mathrm{Zn}$} \\
\hline 1 & 0,6 & 0,8 & 1,2 & 5,0 & 4,8 & 5,5 & 4,7 & 4,9 \\
\hline 2 & 0,5 & 0,6 & 1,6 & 4,7 & 4,5 & 5,1 & 4,5 & 4,4 \\
\hline 3 & 0,5 & 0,8 & 1,0 & 2,9 & 2,9 & 2,8 & 2,9 & 2,7 \\
\hline 4 & 0,5 & 0,7 & 1,0 & 1,9 & 1,9 & 2,1 & 2,1 & 1,8 \\
\hline 5 & 0,9 & 0,9 & 1,1 & 1,5 & 1,5 & 1,7 & 1,6 & 1,6 \\
\hline
\end{tabular}


Numa análise conjunta dos dados dos quadros 2, 3 e 4 vê-se que de um modo geral, os teores de zinco aumentaram significativamente, ao nível de $5 \%$ de probabilidade, quando o tempo de extração passou de 10 para 15 minutos. No entanto a diferença não foi significativa, ao nível de $5 \%$ de probabilidade, entre 15 e 30 minutos de extração.

Analisando-se os dados do quadro 6, verifica-se com 10 minutos de extração, na proporção de $5,0 \mathrm{~g}$ de solo para $50 \mathrm{ml}$ de solução, as quantidades de zinco extraídas pelas soluções de $\mathrm{HC} 1(0,10 \mathrm{~N}$ e 0,05 $\mathrm{N})$, de EDTA a $1 \%$ e de $\mathrm{H}_{2} \mathrm{SO}_{4}(0,05$ e $0,10 \mathrm{~N})$ foram" maiores do que as extraídas pelas demais soluções.

Os dados do quadro 7 mostram novamente a maior capacidade de extração das soluções 'de $\mathrm{HC} 1$, de EDTA a $1 \%$ e de $\mathrm{H}_{2} \mathrm{SO}_{4}$, relativa a das soluções de $\mathrm{MgC1}_{2}$ e de $\mathrm{CH}_{3} \mathrm{COOH}$.

QUADRO 8 - Teores de zinco solúvel (em ppm) nos diferentes solos. Extrações executadas na proporçẫo de $5,0 \mathrm{~g}$ de solo para $50 \mathrm{ml}$ de várias soluções extratoras com 15 minutos de agitação.

\begin{tabular}{|c|c|c|c|c|c|c|c|c|}
\hline \multirow{3}{*}{ 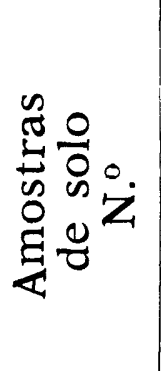 } & \multicolumn{8}{|c|}{ Soluções extratoras } \\
\hline & \multicolumn{2}{|c|}{$\mathrm{MgCl}_{2}$} & \multirow{2}{*}{$\frac{\mid \mathrm{CH}_{3} \mathrm{COOH}}{0,10 \mathrm{~N}}$} & \multirow{2}{*}{$\frac{\mid}{1 \%}$} & \multicolumn{2}{|c|}{$\mathrm{HCl}$} & \multicolumn{2}{|c|}{$\mathrm{H}_{2} \mathrm{SO}_{4}$} \\
\hline & $0,10 \mathrm{~N}$ & $0,50 \mathrm{~N}$ & & & $0,05 \mathrm{~N}$ & $0,10 \mathrm{~N}$ & $0,05 \mathrm{~N}$ & $0,010 \mathrm{~N}$ \\
\hline & \multicolumn{2}{|c|}{ ppm $\mathrm{Zn}$} & \multicolumn{2}{|c|}{ ppm Zn } & \multicolumn{2}{|c|}{$\mathrm{ppm} \mathrm{Zn}$} & \multicolumn{2}{|c|}{ ppm $\mathrm{Zn}$} \\
\hline 1 & 0,5 & 0,8 & 1,3 & 4,3 & 5,4 & 6,0 & 4,9 & 4,7 \\
\hline 2 & 0,3 & 0,7 & 1,7 & 4,0 & 4,9 & 5,4 & 4,7 & 4,5 \\
\hline 3 & 0,4 & 1,0 & 1,2 & 2,1 & 3,0 & 3,1 & 2,8 & 2,8 \\
\hline 4 & 0,3 & 0,8 & 1,2 & 1,6 & 3,0 & 2,5 & 2,0 & 1,8 \\
\hline 5 & 0,5 & 1,1 & 1,3 & 1,1 & 2,6 & 1,8 & 1,6 & 1,8 \\
\hline
\end{tabular}

Os valores apresentados no quadro 8 confirmam os mencionados nos quadros 6 e 7 quanto à capacidade de extração das diversas soluções extratoras.

Numa análise conjunta dos quadros 6,7 e 8 vê-se que as maiores quantidades de zinco foram sempre extraídas com as soluções de $\mathrm{HC1}$, EDTA de $\mathrm{H}_{2} \mathrm{SO}_{4}$, ocorrendo o inverso com as soluções de $\mathrm{CH}_{3} \mathrm{COOH}$ e $\mathrm{MgCl}_{2}$.

A influência do tempo de extração (dados dos quadros 6, 7 e 8 ) foi similar à já mencionada (dados dos quadros 3,4 e 5) isto é, quan- 
do a proporção de pêso (em gramas) de terra para volume (em ml) de solução foi de 2,5: 50. Assim, quando o tempo de extração passou de 10 para 15 minutos, as diferenças de um modo geral foram significativas ao nível de 5\%. Entretanto, quando as extrações foram executadas durante 30 minutos, as diferenças em relação ao tempo de 15 minutos, as diferenças em relação ao tempo de 15 minutos, não foram significativas ao nível de $5 \%$, de um modo geral.

Comparando agora, o conjunto de dados dos quadros 6,7 e $8 \mathrm{com}$ os dos quadros 3,4 e 5 pode-se concluir que as diferenças nas extrações promovidas entre as proporções 2,5: 50 e 5: 50, de pêso de terra (em gramas) para volume (em $\mathrm{ml}$ ) de solução extratora, foram significativas ao nível de $5 \%$ de probabilidade. Isso significa que as extrações na proporção de 2,5: 50 foram maiores do que as conduzidas na proporção de $5: 50$.

\section{CONCLUSÕES}

a) O método baseado na fotometria de absorção atômica de determinação do zinco extraído do solo com diversas soluções, apresentou precisão satisfatória; e a exatidão, avaliada através de ensaio de recuperação, quando foi empregada solução de HC1 $0,10 \mathrm{~N}$ como extrator de zinco, foi também satisfatória.

b) A extração do zinco de cinco amostras de solos com soluções de $\mathrm{HC1} 0,05$ e $0,1 \mathrm{~N}, \mathrm{H}_{2} \mathrm{SO}_{4} 0,05$ e $0,10 \mathrm{~N}$ a $1 \%$, ácido acético $0,10 \mathrm{Ne} \mathrm{MgC1}_{2}$ 0,10 e 0,50 N, mostrou que as soluções de ácidos inorgânicos e a da EDTA são mais eficientes que as demais.

c) A proporção de $2,5 \mathrm{~g}$ de solo para $50 \mathrm{ml}$ de solução extratora permitem uma extração mais elevada de zinco do que a de 5,0 g de solo e $50 \mathrm{ml}$ de solução extratora.

d) O tempo de extração de 15 minutos forneceu dados mais elevados do que o de 10 minutos; no entanto o tempo de 30 minutos forneceu resultados iguais aos obtidos com 15 minutos, isto é, as diferenças não foram significativas ao nível de $5 \%$ de probabilidade.

\section{SUMMARY}

\section{EXTRACTABLE ZINC IN SOILS}

Eight extractants $\left(0.10\right.$ and $0.50 \mathrm{~N} \mathrm{MgC1}_{2} ; 0.10 \mathrm{~N} \mathrm{CH}_{3} \mathrm{COOH}$; $1 \% \mathrm{Na}_{2}$ EDTA; 0.05 and $0.10 \mathrm{~N} \mathrm{HC} 1$; and 0.05 and $0.10 \mathrm{~N} \mathrm{H}_{2} \mathrm{SO}_{4}$ ) were tested in five soil samples using two solution: soil ratio $(50 \mathrm{ml}$ : 2.50 grams and $50 \mathrm{ml}: 5.0$ grams) and three lenght of extraction periods $(10,15$ and 30 minutes) for the extraction of zinc. The zinc concentration of the extractants was determined by atomic absorption photometry. 
The data obtained showed that $1 \% \mathrm{Na}_{2}$ EDTA, 0.05 and $0.10 \mathrm{~N}$ $\mathrm{HC} 1$ and 0.05 and $0.10 \mathrm{~N} \mathrm{H}_{2} \mathrm{SO}_{4}$ solutions extract more zinc tham $\mathrm{MgCl}_{2}$ and $\mathrm{CH}_{3} \mathrm{COOH}$ solutions.

The solution-soil ratio showed significant effects on the zinc extraction. The length of extraction period showed also significante effects only whem the 15 minutes was compared to the 10 minutes pericd of extraction.

\section{LITERATURA CITADA}

BROWN, A. L., B. A. KRANTZ, \& P. E. MARTIN $1962-$ Plant uptake and fate of soil-applied zinc. Soil Sci. Soc. Amer. Proc., Vol. 26(2):167-170.

BROWN, A. L., B. A. KRANTZ, \& P. E. MARTIN, 1964 - The residual effect of zinc applied to soils. Soil Sci. Amer. Proc. Vol. 28(2):236-238.

BROWN, A. L., J. QUINCK \& J. L. EDDINGS, 1971a. A comparison of analytical methods. Soil Sci. Soc. Amer. Proc. 35:105-107.

CHAPMAN, H. D., 1966 - Zinc. 'Em: "Diagnostic Criteria for Plants and Soils". Editado por H. D. Chapman. University of California. Division of Agricultural Sciences. pp. 494-499.

HODGSON, J. F., 1963 - Chemistry of the Micronutrient Elements in Soil. Em "Advances in Agronomy". Editado por A. G. Norman. Academic Press Inc. 15:119-159.

JACKSON, M. L., 1958 - Soil Chemical Analysis. Prentice Hall Inc. Englewood Cliffs. N. J. 498 pp.

MARTENS, D. C., 1968 - Plant availability of extractable boron, copper, and zinc as related to selected soil properties. Soil Sci. 106(1):23-28.

MARTENS, D. C. \& G. CHESTERS, 1967 - Comparison of chemical tests for estimation of the availability of soil zinc. J. Sci. Fd. Agric. Vol. 18, may.

SORENSEN, R. C., D. D. DELSLIGLE \& KNUDSEN, 1971 - Extraction of Zn $\mathrm{Fe}$, and $\mathrm{Mn}$ from soil with $0,1 \mathrm{~N}$ hydrochloric and as affected by soil properties solution soil ratio and lenght of extraction period. Soil Sci. 111:352-359.

STEWART, J. A. \& K. C., BERGER, 1965 - Estimation of available soil zinc. using magnesium chloride as extractant. Soil Sci. 100:244-250.

VIETS. JR., F. G. \& L. C. BOAWN, 1965 - Zinc. Em: Methods of Soil Analysis. Part. 2. Copyright by the American Society of Agronomy, Inc. Publisher. Medison, Wisconsin, U.S.A. (1090-1101). 
. 